\title{
Tempo utilizando computador como discriminador de obesidade, sedentarismo e fatores de risco cardiovascular em universitários
}

\section{Time using a computer as a discriminator of obesity, sedentarism and cardiovascular risk factors in university students}

\author{
Heloísa Silva Guerra1,2 (D) heloisasguerra@gmail.com \\ Adriana Vieira Macedo Brugnoli, (1) adrianavieiramacedo@hotmail.com \\ Raiana Rodrigues Costa Melo1,2 (1) raianarodrigues@unirv.edu.br \\ Emílio Hideyuki Moriguchi' ${ }^{1}$ (D) emilio.moriguchi@gmail.com \\ Marcos Pascoal Pattussi' (1) mppattussi@unisinos.br \\ Juvenal Soares Dias da Costa ${ }^{1,3}$ (1) episoares@terra.com.br
}

\section{RESUMO}

Introdução: Os universitários apresentam maior risco de adotar o comportamento sedentário em virtude da própria rotina, a qual requer muito tempo dedicado às aulas e a utilização frequente do computador para os estudos. Essa situação tem se tornado preocupante porque o comportamento sedentário tem sido associado a desfechos adversos em saúde, como mortalidade e doenças crônicas não transmissíveis.

Objetivo: Este estudo teve como objetivo analisar o tempo despendido com o uso do computador como discriminador da obesidade, do sedentarismo e de fatores de risco cardiovascular em universitários.

Método: Trata-se de estudo transversal realizado com 2.275 acadêmicos de cursos da área da saúde de uma fundação pública do estado de Goiás. Os dados foram obtidos por meio da aplicação de um questionário composto por variáveis sociodemográficas, relativas ao curso e a aspectos comportamentais e de saúde. A variável de desfecho foi o tempo utilizando o computador (TC). Identificaram-se o poder discriminatório e os pontos de corte do TC para os desfechos de interesse por meio das curvas Receiver Operating Characteristic (ROC) com IC95\%.

Resultado: O tempo médio usando o computador para estudos, trabalho ou lazer foi de 3,90 horas/dia para mulheres e 3,82 horas/dia para homens. A área sob a curva ROC entre o TC e o sedentarismo foi de 0,54 (IC95\% 0,51-0,58) para mulheres e 0,56 (IC95\% 0,50-0,63) para homens. Já para a hipertensão arterial sistêmica (HAS), foi de 0,57 (IC95\% 0,50-0,64) para mulheres. Os melhores pontos de corte relacionados a essas condições foram 3,5 e 4,5 horas, respectivamente.

Conclusão: O TC apresentou boa capacidade preditiva para discriminar o sedentarismo e a HAS entre universitários. Sugere-se que a diminuição do TC e sua substituição por atividades ativas possam contribuir para a melhoria do perfil de saúde e a qualidade de vida dos acadêmicos.

Palavras-chave: Comportamento Sedentário; Estilo de Vida; Saúde do Estudante.

\begin{abstract}
Introduction: University students are at greater risk of adopting a sedentary behavior due to their routine, with a large amount of time being dedicated to classes and studying in front of a computer. This situation has become a matter of concern, because sedentary behavior has been associated with adverse health outcomes such as mortality and chronic non-communicable diseases.
\end{abstract}

Objective: To analyze the time spent using the computer as a discriminator of obesity, sedentary lifestyle and cardiovascular risk factors in University students.

Method: This was a cross-sectional study with 2,275 students from courses in the health area of a public institution in Goiás. Data were obtained by applying a questionnaire consisting of sociodemographic, course-related, behavioral and health variables. The outcome variable was time using the computer (TC). The discriminatory power and the cutoff points of time using the computer for the outcomes of interest were identified using the Receiver Operating Characteristic (ROC) curves with 95\%Cl.

Result: The mean time spent using the computer for studying, working, or leisure was 3.90 hours/day for women and 3.82 hours/day for men. The area under the ROC curve between TC and sedentary lifestyle was 0.54 (95\% Cl 0.51-0.58) for women and 0.56 (95\% Cl 0.50 - 0.63) for men. As for systemic arterial hypertension (SAH) it was 0.57 (95\% Cl 0.50-0.64) for women. The best cutoff points related to these conditions were 3.5 and 4.5 hours, respectively.

Conclusion: The TC showed good predictive capacity to discriminate a sedentary lifestyle and SAH among University students. It is suggested that the reduction of TC and its replacement by active activities can contribute to improving the health profile and quality of life of students.

Keywords: Sedentary Behavior; Lifestyle; Student Health.

1 Universidade do Vale do Rio dos Sinos, São Leopoldo, Rio Grande do Sul, Brasil.

2 Universidade de Rio Verde, Aparecida de Goiânia, Goiás, Brasil.

${ }^{3}$ Universidade Federal de Pelotas, Pelotas, Rio Grande do Sul, Brasil.

Editora-chefe: Rosiane Viana Zuza Diniz. $\quad$ | Editor associado: Antonio da Silva Menezes Junior.

Recebido em 10/09/21; Aceito em 04/11/21. | Avaliado pelo processo de double blind review. 


\section{INTRODUÇÃO}

O comportamento sedentário (CS) tem sido investigado em diferentes contextos devido à relação negativa que apresenta com a saúde. Quando considerado excessivo, mostra-se associado a desfechos como mortalidade, doença cardiovascular (DCV), diabetes tipo 2 e síndrome metabólica'. Grandes períodos despendidos em atividades sedentárias estão associados a incrementos no risco de obesidade e menor tempo de atividade física (AF) ${ }^{2,3}$.

Os universitários são um público que apresenta maior risco de adotar o CS em virtude da própria rotina, a qual requer muito tempo despendido nas aulas e a utilização frequente do computador para os estudos 4 . Nos estudos sobre CS, é grande a variabilidade dos métodos de mensuração, bem como diferentes pontos de corte adotados para classificar indivíduos com maior ou menor CS e os tipos de comportamentos avaliados. No entanto, uma revisão sistemática cujo objetivo foi sintetizar as evidências sobre os níveis de CS em estudantes universitários apontou que o uso do computador apresentou prevalência significativamente maior em relação a outras modalidades de tempo de tela ${ }^{5}$.

A mudança de vida iniciada com o ingresso na universidade tem sido relacionada a uma diminuição na prática de $A F$ e ao aumento do $\mathrm{CS}^{6,7}$. Além disso, sabe-se que uma proporção considerável de universitários apresenta níveis mais altos de CS em comparação com a população adulta em geral ${ }^{5}$.

Dentre os eventos prejudiciais à saúde, as doenças crônicas não transmissíveis (DCNT) têm papel de destaque, uma vez que sua ocorrência tem aumentado, constituindo o grupo de doenças de maior magnitude no mundo e a principal causa de óbitos em adultos, especialmente em países de baixa e média rendas ${ }^{8}$.

Os principais fatores de risco para DCNT são hipertensão arterial sistêmica (HAS), diabetes mellitus (DM), dislipidemias, hábitos alimentares inadequados, sedentarismo, tabagismo e obesidade ${ }^{9,10}$. Muitos desses fatores são passíveis de surgir ou ser agravados durante o período de graduação, pois o ingresso na universidade pode implicar mudança do estilo de vida com a adoção de hábitos não saudáveis ${ }^{9}$.

Dados recentes da Pesquisa Nacional de Saúde apontaram que cerca de 96 milhões de brasileiros acima de 18 anos apresentavam excesso de peso, correspondendo a $60,3 \%$ da população. Além disso, 39,2\% apresentavam HAS e 15,9\% diabetes ${ }^{11}$.

Estabelecer a relação quantitativa entre o tempo utilizando o computador (TC) e determinadas condições, como obesidade, sedentarismo e fatores de risco cardiovascular, pode contribuir para a implementação de medidas que desestimulem esses comportamentos e incentivem os universitários à adotar práticas mais ativas, de modo a prevenir os referidos agravos.
Diante disso, o objetivo deste estudo foi analisar o tempo despendido usando o computador como discriminador da obesidade, do sedentarismo e de fatores de risco cardiovascular em universitários.

\section{MÉTODOS}

Realizou-se estudo de delineamento transversal, de base escolar, em uma amostra do tipo censo, com estudantes universitários vinculados a uma fundação pública municipal localizada em Goiás, no Brasil, no ano de 2018.

Incluíram-se estudantes de ambos os sexos e com idade igual ou superior a 18 anos, e excluíram-se aqueles que possuíam algum tipo de deficiência física e com idade igual ou superior a 60 anos. Para o cálculo do tamanho da amostra, considerou-se uma prevalência estimada de 50\%, levando-se em conta a inclusão de diferentes informações relacionadas à saúde e erro de 3 pontos percentuais. Foram acrescentados $10 \%$ para possíveis perdas ou recusas e 15\% para controle de fatores de confusão. Assim, estimou-se a necessidade de incluir 953 indivíduos.

A população do estudo constou de todos os acadêmicos de graduação dos cursos de saúde da instituição (Educação Física, Enfermagem, Farmácia, Fisioterapia, Medicina e Odontologia), distribuídos em três campi (Aparecida de Goiânia, Goianésia e Rio Verde), num total de 2.658 alunos.

Para a operacionalização da coleta de dados, utilizouse um questionário impresso, pré-testado, padronizado e autoaplicável, composto por variáveis sociodemográficas, relativas ao curso e a aspectos comportamentais e de saúde.

O TC foi avaliado por meio de uma pergunta sobre o tempo despendido, em horas e minutos, em um dia normal da semana, utilizando o computador para trabalho, estudo ou lazer. Os minutos do TC foram transformados em horas.

As variáveis demográficas incluíram sexo (feminino; masculino), idade (18-19 anos; 20-24 anos; 25-29 anos; 30 anos ou mais), cor da pele autorreferida (branca; parda; preta; outras) e situação conjugal (com companheiro; sem companheiro). A variável socioeconômica foi a classificação econômica da Associação Brasileira de Empresas de Pesquisa (Abep), cujos escores foram organizados em categorias $(A ; B$; C/D/E). Estabeleceu-se a classificação da Abep a partir da posse e quantidade de alguns bens de consumo, da escolaridade do chefe da família e do acesso a determinados serviços públicos ${ }^{12}$.

As variáveis relacionadas às características discentes foram o curso (Medicina; outros) e o período (do primeiro ao sexto; do sétimo ao $12^{\circ}$ ). Consideraram-se os seguintes hábitos de vida: tabagismo (não fumantes; ex-fumantes; fumante atual) uso de drogas ilícitas nos últimos 30 dias (uso; não uso), uso de álcool (uso; não uso) e prática de AF. Quanto à AF, coletaram-se os 
dados por meio do International Physical Activity Questionnaire $(\text { IPAQ })^{13}$. Nesse item, os indivíduos foram assim categorizados: sedentário (não realiza nenhuma $\mathrm{AF}$ por pelo menos dez minutos contínuos durante a semana), insuficientemente ativo (pratica AF por pelo menos dez minutos contínuos por semana, porém de maneira insuficiente para ser classificado como ativo), ativo (cumpre as seguintes recomendações: 1 . AF vigorosa - $\geq 3$ dias/semana e $\geq 20$ minutos/sessão; 2 . moderada ou caminhada $-\geq 5$ dias/semana e $\geq 30$ minutos/sessão; 3. qualquer atividade somada $-\geq 5$ dias/semana e $\geq 150 \mathrm{~min} /$ semana) e muito ativo (cumpre as seguintes recomendações: 1. atividade vigorosa $-\geq 5$ dias/semana e $\geq 30 \mathrm{~min} /$ sessão; 2 . vigorosa - $\geq 3$ dias/semana e $\geq 20 \mathrm{~min} /$ sessão + moderada e/ou caminhada $\geq 5$ dias/semana e $\geq 30 \mathrm{~min} /$ sessão).

Por fim, as informações relacionadas à saúde foram o Índice de Massa Corporal (IMC) e a presença de doenças que aumentavam o risco cardiovascular. O IMC foi calculado a partir do peso e da altura referidos pelo entrevistado e categorizado de acordo com os pontos de corte recomendados pela Organização Mundial da Saúde (OMS), sendo IMC até 24,99 (peso adequado), > 25,00 e $<29,99$ (sobrepeso) e $\geq$ 30 (obesidade) $^{14}$. A presença de doenças foi considerada a partir da declaração autorreferida, com diagnóstico prévio realizado por médico, das seguintes condições: DM, HAS e hipercolesterolemia.

A estruturação do banco de dados foi feita no programa EpiData 3.1 com dupla entrada para correção de inconsistências. Analisaram-se os dados por meio software Statistical Package for the Social Sciences (SPSS) versão 22.0. Na análise descritiva, calcularam-se os valores de média e desvio padrão, frequências absoluta e relativa e medidas de associação para variáveis categóricas (teste qui-quadrado) com $p<0,05$. Utilizou-se estratégia de estratificação por sexo em virtude das diferenças observadas na literatura quanto aos fatores associados ao CS entre homens e mulheres, a depender do tipo de atividade considerada $^{15,16}$.

A capacidade discriminatória, a sensibilidade e a especificidade do TC para avaliar a presença das condições estabelecidas foram realizadas a partir da análise das curvas Receiver Operating Characteristic (ROC). A área sob a curva (ASC) determinou a capacidade discriminatória do indicador para presença ou ausência de obesidade, sedentarismo e fatores de risco para doenças cardiovasculares. As áreas com valores > 0,50 e limite inferior do IC95\% > 0,50 foram consideradas como discriminatórias para a estimativa de pontos de corte ${ }^{17}$. Estimaram-se os níveis de sensibilidade e especificidade para os pontos de corte, e os pontos que melhor discriminaram a presença das condições já citadas foram aqueles que apresentaram equilíbrio entre essas duas características $^{18}$.

Adotaram-se as Diretrizes e Normas Regulamentadoras de Pesquisa Envolvendo Seres Humanos, conforme a Resolução n 466 do Conselho Nacional de Saúde. Os Comitês de Ética da Universidade do Vale do Rio dos Sinos - Unisinos (Parecer $n^{\circ}$ 2.892.764) e Universidade de Rio Verde - UniRV (Parecer $n^{\circ}$ 2.905.704) aprovaram o estudo.

\section{RESULTADOS}

Participaram do estudo 2.275 universitários. O tempo médio despendido usando o computador para estudos, trabalho ou lazer foi de 3,90 (DP $\pm 2,98$ ) horas/dia para mulheres e 3,82 (DP $\pm 2,96$ ) horas/dia para homens.

A análise estratificada apontou diferenças entre os acadêmicos quanto à faixa etária, à cor da pele, à classe econômica, ao curso, ao hábito de fumar, ao uso de bebida alcoólica, à $A F$, ao IMC e à presença de hipercolesterolemia (Tabela 1). Encontrou-se uma maior proporção de indivíduos do sexo feminino na faixa etária de 20 a 24 anos (70,7\%), cor da pele branca (58\%), não fumantes (90,3\%), ativos fisicamente $(42,2 \%)$, sem obesidade $(96,0 \%)$ e que não apresentavam hipercolesterolemia (81,5\%). Entre os participantes do sexo masculino, foi observada maior prevalência de indivíduos inseridos na classe econômica A (53,5\%), no curso de Medicina $(74,2 \%)$ e referindo uso de bebida alcoólica $(79,1 \%)$ (Tabela 1).

A partir da análise realizada, identificaram-se os valores das ASC do TC como discriminador para a presença de obesidade, sedentarismo e fatores de risco cardiovascular em universitários. Verificou-se que o TC foi um bom discriminador de sedentarismo entre mulheres e homens, e da presença de HAS entre mulheres. Para as demais condições, o valor de $p$ não se mostrou estatisticamente significante (Tabela 2).

Quando se analisaram os pontos de corte com maior acurácia e valores de sensibilidade e especificidade da relação entre oTC e o sedentarismo, observou-se que, entre as mulheres, o ponto de corte que melhor discriminou essa condição foi de mais de 3,5 horas/dia (sensibilidade $=53 \%$ e especificidade $=$ 53\%) (Gráfico 1).

Entre os homens, o ponto de corte que melhor discriminou a presença do sedentarismo também foi $\geq 3,5$ horas/dia (sensibilidade $=57 \%$ e especificidade $=55 \%$ ) (Gráfico 2$)$.

O Gráfico 3 apresenta a área sob a curva ROC entre o TC e a presença de HAS no sexo feminino, e o melhor ponto de corte encontrado foi de $\geq 4,5$ horas/dia (sensibilidade $=45 \%$ e especificidade $=66 \%$ ). 
Tabela 1. Características sociodemográficas, discentes, hábitos de vida e saúde dos universitários. Goiás, 2018.

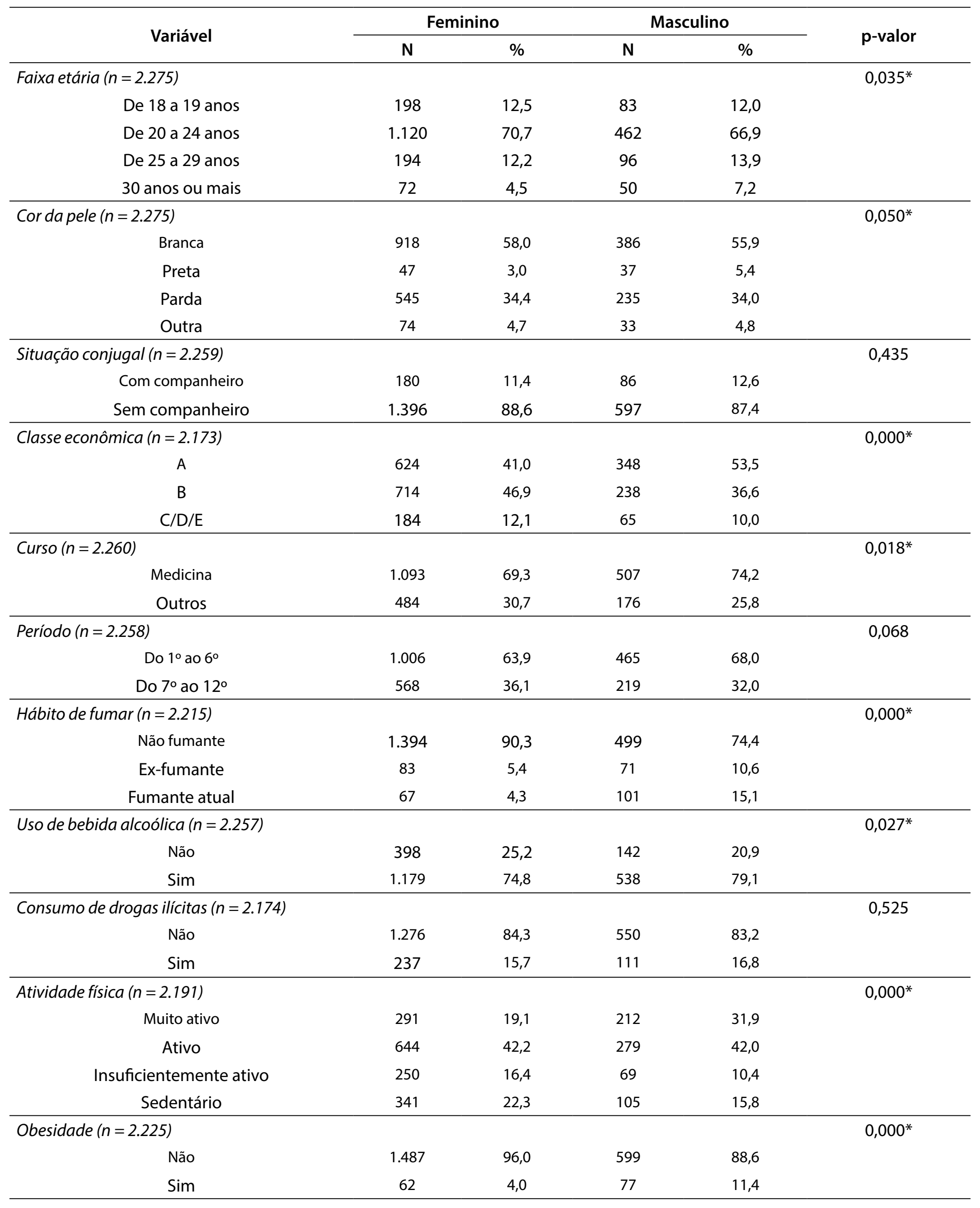


Tabela 1. (Continuação) Características sociodemográficas, discentes, hábitos de vida e saúde dos universitários. Goiás, 2018.

\begin{tabular}{|c|c|c|c|c|c|}
\hline \multirow{2}{*}{ Variável } & \multicolumn{2}{|c|}{ Feminino } & \multicolumn{2}{|c|}{ Masculino } & \multirow{2}{*}{ p-valor } \\
\hline & $\mathrm{N}$ & $\%$ & $\mathrm{~N}$ & $\%$ & \\
\hline Hipertensão arterial $(n=2.244)$ & & & & & 0,108 \\
\hline Sim & 73 & 4,7 & 21 & 3,1 & \\
\hline Diabetes mellitus ( $n=2.231$ ) & & & & & 0,255 \\
\hline Sim & 76 & 4,9 & 41 & 6,1 & \\
\hline Hipercolesterolemia $(n=2.222)$ & & & & & $0,013^{*}$ \\
\hline Não & 1.267 & 81,5 & 513 & 76,8 & \\
\hline Sim & 287 & 18,5 & 155 & 23,2 & \\
\hline
\end{tabular}

Teste qui-quadrado de Pearson - *p $\leq 0,05$.

Fonte: Elaborada pelos autores.

Tabela 2. Área sob a curva ROC e IC95\% doTC como discriminador da obesidade, do sedentarismo e de fatores de risco cardiovascular em universitários. Goiás, 2018.

\begin{tabular}{ccccc}
\hline \multirow{2}{*}{ Variáveis } & \multicolumn{2}{c}{ Sexo feminino } & \multicolumn{2}{c}{ Sexo masculino } \\
\cline { 2 - 5 } & ASC (IC95\%) & p-valor & ASC (IC95\%) & p-valor \\
\hline Obesidade & $0,560(0,494-0,627)$ & 0,108 & $0,535(0,457-0,613)$ & 0,325 \\
Sedentarismo & $0,542(0,507-0,578)$ & $0,018^{*}$ & $0,563(0,501-0,627)$ & $0,041^{*}$ \\
HAS & $0,574(0,506-0,643)$ & $0,032^{*}$ & $0,499(0,387-0,611)$ & 0,987 \\
Diabetes & $0,509(0,445-0,572)$ & 0,794 & $0,534(0,436-0,632)$ & 0,487 \\
Hipercolesterolemia & $0,520(0,483-0,557)$ & 0,287 & $0,467(0,413-0,520)$ & 0,214 \\
\hline
\end{tabular}

ASC = área sob a curva; ROC = Receiver Operating Characteristic; IC95\% = intervalo de confiança de 95\%; HAS = hipertensão arterial sistêmica; ${ }^{*} \mathrm{p}<0,05$.

Fonte: Elaborada pelos autores.

Gráfico 1.Área sob a curva (ROC) com ponto de corte para o tempo de computador como discriminador do sedentarismo em mulheres universitárias de Goiás, Brasil, 2018.

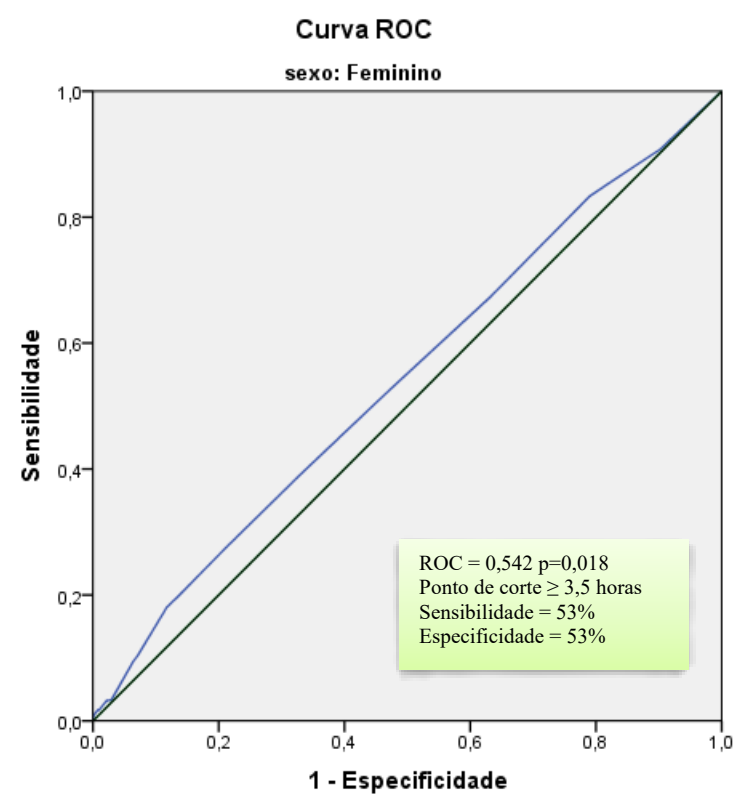

Fonte: Elaborado pelos autores.
Gráfico 2. Área sob a curva (ROC) com ponto de corte para o tempo de computador como discriminador do sedentarismo em homens universitários de Goiás, Brasil, 2018.

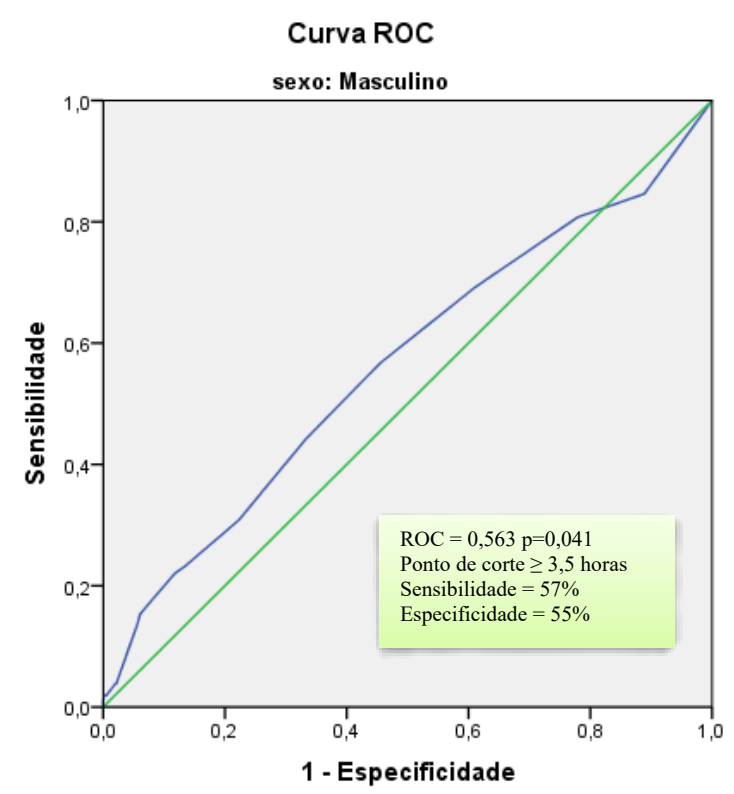

Fonte: Elaborado pelos autores. 
Gráfico 3. Área sob a curva (ROC) com ponto de corte para o tempo de computador como discriminador da hipertensão arterial sistêmica em mulheres universitárias de Goiás, Brasil, 2018.

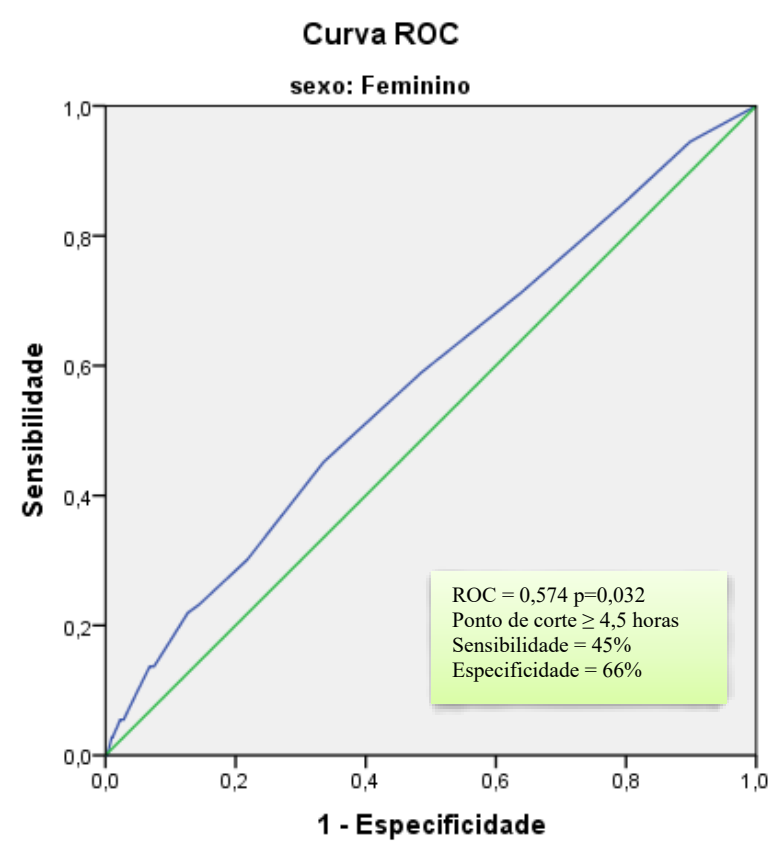

Fonte: Elaborado pelos autores.

\section{DISCUSSÃO}

O presente estudo buscou demonstrar o poder discriminatório e o ponto de corte do TC em relação a algumas condições, como obesidade, sedentarismo e fatores de risco para DCV em estudantes universitários. Poucos estudos na literatura utilizaram o CS ou um de seus componentes como discriminador de condições em saúde na população universitária, ao contrário de outros grupos como $\operatorname{crianças~}^{19}$, adolescentes ${ }^{20}$ e idosos ${ }^{21,22}$.

As análises identificaram os valores que apresentaram o melhor equilíbrio entre sensibilidade e especificidade para discriminar a presença de sedentarismo e HAS entre os graduandos. Os dados mostraram que a capacidade preditiva do TC para discriminar a presença de sedentarismo foi maior entre homens do que entre mulheres, já que os valores da ASC para as mulheres foram mais baixos. Para a HAS, apenas entre as mulheres o TC apresentou poder discriminatório.

Na literatura, foi possível encontrar diferenças quanto ao CS quando se compararam homens e mulheres, pois, a depender do domínio considerado, um sexo podia se sobrepor ao outro. Pesquisa com dados de oito países latino-americanos apontou que os homens apresentaram maior CS do que as mulheres em uso de computador, videogame, leitura e deslocamento no transporte, independentemente da idade ${ }^{15}$. No entanto, no presente estudo, a média de TC entre os universitários dos sexos masculino e feminino foi equivalente, talvez porque as atividades desenvolvidas diariamente que implicavam o uso desse dispositivo fossem semelhantes.

Em relação aos pontos de corte, é importante ressaltar que a literatura ainda não apresenta consenso sobre a quantidade de tempo a partir do qual o excesso de CS poderia ser prejudicial à saúde, sendo possível encontrar tempos variando de duas horas até dez horas ou mais por dia $^{23}$. Um estudo que buscou observar a relação entre o CS, as doenças cardiovasculares e a mortalidade em adultos apontou que duas horas/dia de tempo de tela estavam associadas a um aumento de 5\% (HR 1,05; IC95\% 1,01-1,09) em eventos cardiovasculares ${ }^{24}$.

Outros estudos realizados no Brasil tentaram identificar o ponto de corte mais adequado para a população universitária. Em Minas Gerais, os autores buscaram selecionar os melhores pontos de corte do tempo sentado como discriminador da ausência de morbidades referidas, como obesidade, excesso de peso, HAS, colesterol elevado e glicemia elevada. Eles concluíram que o melhor ponto de corte para ausência de HAS foi de seis horas ${ }^{25}$. Já na Bahia, um estudo realizado com acadêmicas de Enfermagem apontou o tempo sentado por oito horas ou mais por dia como o que melhor discriminou a obesidade abdominal entre as estudantes ${ }^{26}$. Desta forma, essas pesquisas encontraram pontos de corte bem acima do evidenciado na análise do presente estudo.

Uma revisão sistemática da literatura sobre CS demonstrou que os universitários gastavam em média 7,29 horas por dia sentados, tempo maior que a média de adultos jovens em países de alta renda. Nessa mesma revisão, a síntese dos dados acerca do tempo médio utilizando o computador foi de 2,91 (IC95\% 2,32-3,5) horas por dia, valor inferior ao encontrado no presente estudo, tanto para os homens quanto para as mulheres ${ }^{5}$.

Quando se considera que é grande a possibilidade de os universitários assumirem ocupações futuras com exposição a longos períodos de tempo sentado durante o dia de trabalho, os dados tornam-se preocupantes, uma vez que a tendência desse tempo é aumentar. Um estudo realizado com adultos nos Estados Unidos certificou que o tempo sedentário aumentou na última década em todos os subgrupos analisados, considerando idade, sexo, nível de escolaridade, raça e IMC ${ }^{27}$.

A literatura apresenta uma associação negativa do CS com a $\mathrm{AF}^{3}$. Universitários fisicamente ativos tendem a dedicar menos tempo a comportamentos sedentários ${ }^{28}$. O sedentarismo pode trazer vários prejuízos à saúde, como predispor o indivíduo ao excesso de peso e à obesidade, que, por sua vez, estão relacionados a outros problemas. Estudos de casos e controles com universitários de Bangladesh apontaram que os não praticantes de nenhuma AF apresentaram uma 
chance 3,2 vezes maior de serem obesos ou terem sobrepeso quando comparados aos ativos fisicamente (OR = 3,2; IC95\%: $1,0-9,9 ; p<0,05)^{29}$.

No presente estudo, embora a análise estratificada não tenha apresentado grande diferença, tanto mulheres quanto os homens passaram quase um turno de seu dia diante de um computador, sem levar em consideração os outros componentes do CS, o que representou quase $25 \%$ do tempo de vigília. Esse fato chamou a atenção e tornou-se ainda mais preocupante, pois, em virtude da situação sanitária mundial causada pela pandemia da coronavirus disease 2019 (Covid-19), a rotina da maioria das pessoas foi modificada, tendo impactado em especial o âmbito estudantil, com aulas sendo ministradas na modalidade a distância, o que exigiu um tempo maior que o habitual, por parte dos estudantes, diante de dispositivos eletrônicos como o computador e smartphones.

Uma análise realizada com universitários da Espanha mostrou que o tempo sentado aumentou durante o período de lockdown entre todos os grupos de estudantes avaliados, refletindo as consequências trazidas pelo período de restrição em função da pandemia ${ }^{30}$. No Brasil, um inquérito que investigou vários comportamentos da população brasileira durante a pandemia de Covid-19 demonstrou um aumento do tempo médio assistindo à TV e usando o computador ou tablet, com um incremento de 1 hora e 30 minutos em relação ao tempo gasto com essas atividades antes da pandemia. O maior tempo médio de uso do computador foi entre adultos jovens de 18 a 29 anos, com cerca de 7 horas e 15 minutos, representando um aumento de quase três horas sobre o tempo de uso antes da pandemia ${ }^{31}$.

Como pontos fortes deste estudo, destacam-se a representatividade da amostra de universitários de uma região do país ainda pouco explorada, a rigorosa condução do estudo, bem como a relevância do conhecimento sobre um componente do CS muito presente nesse público e sua relação com determinadas condições no âmbito da saúde. Além disso, são poucos os estudos que fizeram esse tipo de análise, principalmente no Brasil.

Entre as limitações, estão a avaliação do TC por meio de questionário e a utilização de medidas autorreferidas para a obtenção das informações sobre morbidades. Entretanto, esse tipo de medida tem sido frequente em estudos epidemiológicos ${ }^{3,32}$. Convém ressaltar que as informações foram coletadas antes da pandemia da Covid-19 e que, em virtude das medidas adotadas para enfrentamento da doença, os achados podem ter sido modificados para pior. Outra limitação diz respeito ao delineamento transversal, que exige cautela na interpretação dos achados, já que estudos longitudinais ou experimentais seriam mais adequados para apresentar a relação entre TC e a presença de determinadas condições.

\section{CONCLUSÃO}

O TC apresentou uma boa capacidade de discriminar a presença de sedentarismo em universitários e de HAS entre estudantes do sexo feminino. Os pontos de corte que melhor discriminaram essas condições foram 3,5 e 4,5 horas, respectivamente.

Ao se refletir sobre os achados, sugere-se que diminuir o TC e substituí-lo por atividades ativas pode contribuir para melhorar o perfil de saúde e a qualidade de vida dos universitários, funcionando como abordagem preventiva de doenças cardiometabólicas e sedentarismo. Os dados podem sugerir que estudos epidemiológicos futuros em estudantes universitários sobre o tempo de tela devem dispensar mais atenção ao tempo de uso do computador.

\section{CONTRIBUIÇÃO DOS AUTORES}

Heloísa Silva Guerra participou da concepção do projeto, da coleta, análise e interpretação dos dados, da redação do manuscrito e da revisão crítica do estudo. Adriana Vieira Macedo Brugnoli e Raiana Rodrigues Costa Melo participaram da coleta e interpretação dos dados, e da revisão crítica do estudo. Emílio Hideyuki Moriguchi participou da interpretação dos dados e da revisão crítica do estudo. Marcos Pascoal Pattussi participou da concepção do projeto, da interpretação dos dados e da revisão crítica do estudo. Juvenal Soares Dias da Costa participou da concepção do projeto, da análise e interpretação dos dados, e da revisão crítica do estudo.

\section{CONFLITO DE INTERESSES}

Declaramos não haver conflito de interesses.

\section{FINANCIAMENTO}

Declaramos não haver financiamento.

\section{REFERÊNCIAS}

1. Rezende LFM, Lopes MR, Rey-López JP, Matsudo VKR, Luiz OC. Sedentary behavior and health outcomes: an overview of systematic reviews. PLoS ONE. 2014;9(8):e105620.

2. Vainsshelboim B, Brennan GM, LoRusso S, Fitzgerald P, Wisniewski KS. Sedentary behavior and physiological health determinants in male and female college students. Physiol Behav. 2019; 204:277-82.

3. Castro O, Bennie J, Vergeer I, Bosselut G, Biddle SJH. Correlates of sedentary behavior in university students: a systematic review. Prev Med. 2018;116:194-202.

4. Cotton $\mathrm{E}$, Prapavessis $\mathrm{H}$. Increasing nonsedentary behaviors in university students using text messages: randomized controlled trial. JMIR Mhealth Uhealth. 2016 Aug 19;4(3):e99.

5. Castro O, Bennie J, Vergeer I, Bosselut G, Biddle S. How sedentary are university students? A systematic review and meta-analysis. Prev Sci. 2020;21(3):332-43.

6. Crombie AP, Ilich JZ, Dutton GR, Panton LB, Abood DA. The freshman weight gain phenomenon revisited. Nutr Rev. 2009;67(2):83-94. 
7. Vella-Zarb RA, Elgar FJ. The "freshman 5": a meta-analysis of weight gain in the freshman year of college. J Am Coll Health. 2009;58(2):161-6.

8. Williams J, Allen L, Wickramasinghe K, Mikkelsen B, Roberts N, Townsend $\mathrm{N}$. A systematic review of associations between non-communicable diseases and socioeconomic status within low-and lower - middle income countries. J Glob Health. 2018;8(2):1-25.

9. Lopes PD, Rezende AAA, Calábria LK. Fatores de risco para doenças crônicas não transmissíveis em universitários. Rev Bras Promoç Saúde. 2017;30(4):1-11.

10. Ribeiro AG, Cotta RMM, Ribeiro SMR. A promoção da saúde e a prevenção integrada dos fatores de risco para doenças cardiovasculares. Cien Saude Colet. 2012;17(1):7-17.

11. Brasil. Pesquisa Nacional de Saúde 2019: atenção primária à saúde e informações antropométricas. Rio de Janeiro: IBGE; 2020.

12. Associação Brasileira de Empresas de Pesquisa. Critério de Classificação Econômica Brasil. CCEB; 2016 [acesso em 10 nov 2020]. Disponível em: https://www.abep.org/criterio-brasil.

13. The IPAQ Group. Guidelines for Data Processing and Analysis of the International Physical Activity Questionnaire. 2005 [acesso em 18 jul 2021]. Disponível em: http://www.ipaq.ki.se.

14. World Health Organization. Obesity: preventing and managing the global epidemic. Report of a WHO consultation. WHO; 1998 [acesso em 18 jul 2021]. Disponível em: https://apps.who.int/iris/handle/10665/63854.

15. Ferrari LM, Werneck AO, Silva DR, Kovalskys I, Gómez G, Rigotti A, et al. Socio-demographic correlates of total and domain-specific sedentary behavior in Latin America: a population-based study. Int J Environ Res Public Health. 2020;17(5587):1-19.

16. Barros MBA, Lima MG, Medina LPB, Szwarcwald CL, Malta DC. Social inequalities in health behaviors among Brazilian adults: National Health Survey, 2013. Int J Equity Health. 2016;15:148-57.

17. Schisterman EF, Faraggi D, Reiser B, Trevisan M. Statistical inference for the area under the receiver operating characteristic curve in the presence of random measurement error. Am J Epidemiol. 2001;154(2):174-9.

18. Lasko TA, Bhagwat JG, Zou KH, Ohno-Machado L. The use of receiver operating characteristic curves in biomedical informatics. J Biomed Inform. 2005;38(5):404-15.

19. Oliveira LC, Ferrari GLM, Araújo TL, Matsudo V. Excesso de peso, obesidade, passos e atividade física de moderada a vigorosa em crianças. Rev Saude Publica. 2017;51(38):1-12.

20. Pitanga FJG, Alves CFA, Pamponet ML, Medina MG, Aquino R. Screen time as discriminator for overweight, obesity and abdominal obesity in adolescents. Rev Bras Cineantropom Desempenho Hum. 2016;18(5):539-47.
21. Silva PAS, Rocha SV, Vasconcelos LRC, Santos CA. Sedentary behavior as discriminator of common mental disorders in elderly. J Bras Psiquiatr. 2017;66(4):183-8.

22. Jesus AS, Rocha SV. Comportamento sedentário como critério discriminador do excesso de peso corporal em idosos. Rev Bras Ativ Fís Saúde. 2018;23:e0030.

23. Franco DC, Ferraz NL, Sousa TF. Sedentary behavior among university students: a systematic review. Rev Bras Cineantropom Desempenho Hum. 2019;21:e56485.

24. Ford ES, Caspersen CJ. Sedentary behaviour and cardiovascular disease: a review of prospective studies. Int J Epidemiol. 2012;41:1338-53.

25. Franco DC. Comportamento sedentário em universitários: estimativas de acurácia, prevalências e fatores associados [dissertação]. Uberaba: Universidade Federal do Triângulo Mineiro; 2019.

26. Mussi FC, Pitanga FJG, Pires CGS. Cumulative sitting time as discriminator of overweight, obesity, abdominal obesity and lipid disorders in nursing university. Rev Bras Cineantropom Desempenho Hum. 2017;19(1):40-9.

27. Du Y, Liu B, Sun Y, Snetselaar LG, Wallace RB, Bao W. Trends in adherence to the physical activity guidelines for Americans for aerobic activity and time spent on sedentary behavior among US adults, 2007 to 2016. JAMA Netw Open. 2019;2(7):e197597.

28. Carballo-Fazanes A, Rico-Díaz J, Barcala-Furelos R, Rey E, RodríguezFernández JE, Varela-Casal $C$, et al. Physical activity habits and determinants, sedentary behaviour and lifestyle in university students. Int J Environ Res Public Health. 2020;17(9):1-15

29. Banna HA, Brazendale K, Hasan M, Khan SI, Sayeed A, Kundu S. Factors associated with overweight and obesity among Bangladeshi university students: a case-control study. J Am Coll Health. 2020;1:1-7.

30. Romero-Blanco A, Rodríguez-Almagro J, Onieva-Zafra MD, ParraFernández ML, Prado-Laguna MC, Hernández-Martínez A. Physical activity and sedentary lifestyle in university students: changes during confinement due to the Covid-19 pandemic. Int J Environ Res Public Health. 2020;17(6567):1-13.

31. Malta DC, Szwarcwald CL, Barros MBA, Gomes CS, Machado IE, Souza Júnior PRB, et al. A pandemia da Covid-19 e as mudanças no estilo de vida dos brasileiros adultos: um estudo transversal, 2020. Epidemiol Serv Saude. 2020;29(4):e20200407.

32. Moreira JPL, Almeida RMVR, Rocha NCS, Luiz RR. Correção da prevalência autorreferida em estudos epidemiológicos com grandes amostras. Cad. Saude Publica. 2016;32(12):e00050816. 